\title{
CONTRIBUCIÓN AL CONOCIMIENTO DEL GÉNERO PLECTANIA (PEZIZALES, SARCOSOMATACEAE) EN MÉXICO
}

\author{
Rosario Medel \\ Y \\ SANTIAGo Chacón \\ Instituto de Ecología, A.C. \\ Apartado postal 63 \\ 91000 Xalapa, Veracruz
}

\section{RESUMEN}

Se discuten los representantes de Plectania previamente conocidos de México: $P$. campylospora, $P$. melaena, $P$. melastoma, $P$. mexicana, $P$. nigrella y $P$. rhytidia f. rhytidia. Plectania rhytidia f. platensis, colectada en el estado de Veracruz, se cita por primera vez del país. Se presenta una clave para las especies mexicanas.

\section{ABSTRACT}

The previously known members of Plectania from Mexico are discussed: Plectania campylospora, P. melaena, P. melastoma, P. mexicana, P. nigrella and P. rhytidia f. rhytidia. Plectania rhytidia f. platensis is reported for the first time for the country from the state of Veracruz. A key for the Mexican species is presented.

\section{INTRODUCCIÓN}

La familia Sarcosomataceae (Pezizales) incluye 14 géneros, la mayoría de distribución boreal y sólo 3 ó 4 se extienden a las porciones calientes de América. Entre estos últimos se encuentra Plectania Fuckel, el cual ha sido poco estudiado en México. Paden (1983), en su revisión de este taxon para el neotrópico, consideró siete especies y dos formas. Las principales referencias que citan y discuten representantes de Plectania en el país son las de Paden (1983) y Pompa-González y Cifuentes (1991). Para el reconocimiento de las especies y datos de su distribución, este trabajo se basó en Korf (1957, 1972), Korf y Weng-Ying (1991), Paden (1983) y Rifai (1968).

Los ejemplares examinados están depositados en el herbario del Instituto de Ecología, A.C. (XAL), salvo un espécimen de Plectania rhytidia f. rhytidia (J. García sin número, marzo 5, de 1988), que se encuentra en el herbario de la Facultad de Biología de la Universidad Veracruzana (XALUV). Los hongos fueron estudiados siguiendo las técnicas rutinarias en micología, elaborando preparaciones temporales en $\mathrm{KOH}$ a $5 \%$, 
solución de Melzer y azul de algodón en lactofenol. Se tomaron fotografías al microscopio electrónico de barrido de las dos formas de $P$. rhytidia descritas en este artículo.

\section{Plectania Fuckel}

Apotecio mediano a grande, turbinado a profundamente cupulado, estipitado o sésil, gregario o solitario, de color negro-grisáceo a casi negro, himenio de color negro a púrpura muy obscuro, la parte externa del receptáculo a veces con gránulos de color anaranjado, margen entero, ondulado a crenado. Las células más externas del apotecio se diferencian en pelos o hifas gruesas, de color café obscuro a negro, extendiéndose varios milímetros fuera del apotecio y sirviéndole como anclaje. Ascas largamente estipitadas, de pared gruesa, octosporadas, inamiloides. Esporas elipsoides, subalantoides o globosas, lisas o con costillas transversales en un solo lado de la espora. Excípulo medular de textura intrincada, excípulo ectal de textura angular (Rifai, 1968; Paden, 1983; Korf y Wen-Ying, 1991).

\section{Las especies de Plectania conocidas de México}

Con el nuevo registro de Plectania rhytidia f. platensis que aquí se presenta, se conocen seis especies y una forma para México. Ya que las especies se encuentran agrupadas en cuatro secciones, según la forma y ornamentación de las esporas (Paden, 1983), a continuación se discuten cada una de ellas de acuerdo con la sección a la cual pertenecen:

Sección Sphaerosporae (esporas lisas y globosas)

P. nigrella (Pers. : Fr.) P. Karst., fue registrada de los estados de México y Guerrero de bosque de pino-encino y mesófilo de montaña (Pompa-González y Cifuentes, 1991). Paden (1983) mencionó que la especie probablemente es de amplia distribución, encontrándose en Norte América, El Caribe, India, Madagascar y Nueva Zelanda.

P. melaena (Fr.) Paden (=Pseudoplectania vogesiaca (Pers.) Fr.) ha sido citada de los estados de Guerrero y Michoacán (Pompa-González y Cifuentes, 1991), donde crece en bosques mesófilo de montaña, de pino-encino y de pino-oyamel. Paden (1983) indicó que esta especie se distribuye en Europa, Norte América y Australia.

\section{Sección Plectania (esporas lisas y elipsoides)}

P. mexicana (Ellis \& Holway) Paden (=Bulgaria mexicana Ellis \& Holway; =Sarcosoma mexicana (Ellis \& Holway) Paden \& Tylutki) fue descrita de Morelos (Paden, 1983; Paden \& Tylutki, 1969). Paden (1983) señaló que la colecta original probablemente provenía de un bosque de Pinus-Quercus, además de considerarla como una especie rara 
Medel y Chacón: Contribución al Conocimiento del Género Plectania en México

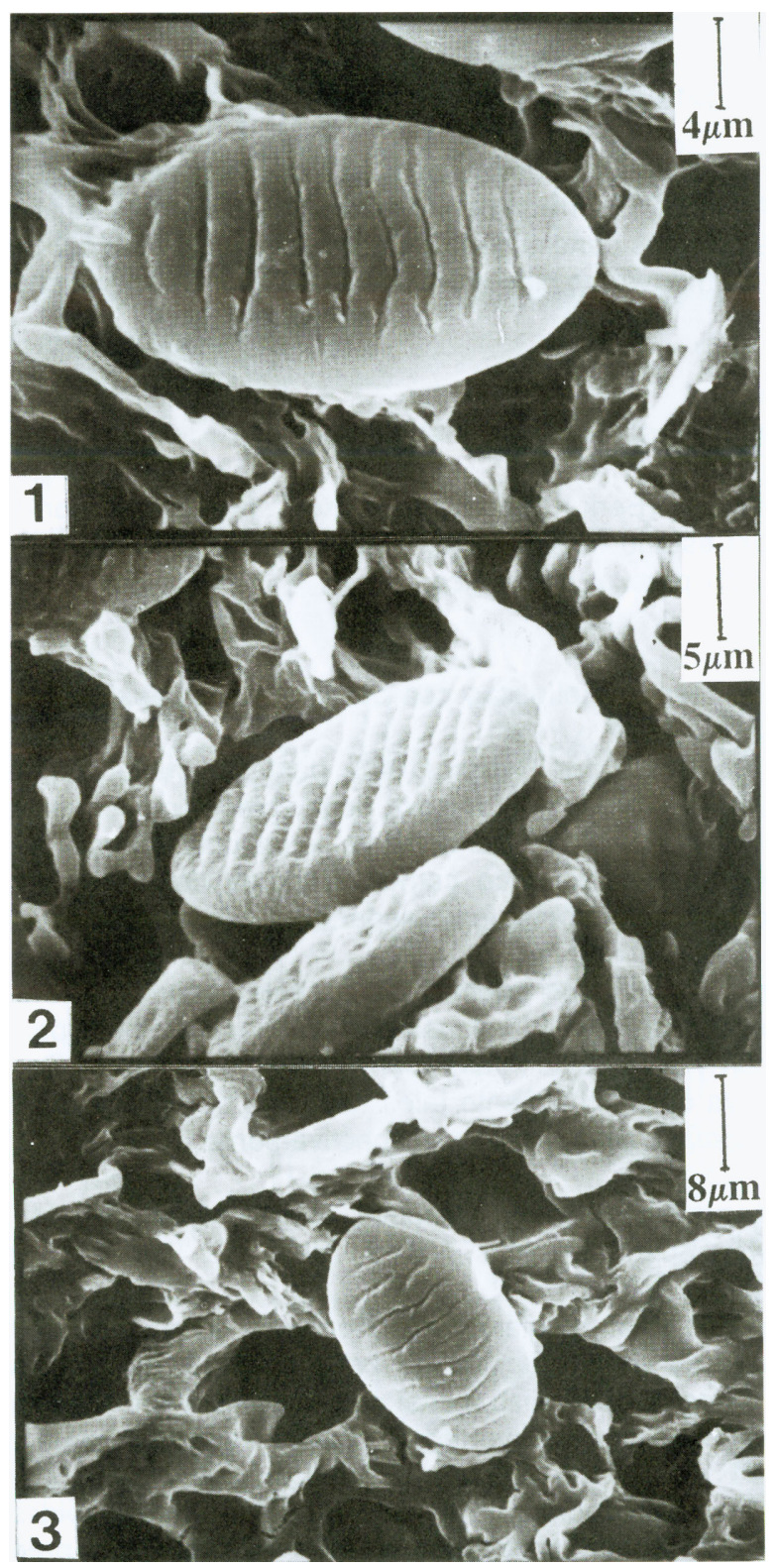

Fig. 1. Plectania rhytidia f. platensis, ascosporas, nótese las estriaciones no anastomosadas. Figs. 2-3. Plectania rhytidia f. rhytidia. 2. ascosporas en vista lateral; 3. ascosporas en vista frontal. 
en México. Sin embargo, más recientemente Frutis y Guzmán (1983) citaron este hongo de Hidalgo, en bosque de abetos, Díaz-Barriga et al. (1988) de Michoacán, y Heredia (1989) de Tamaulipas; en los dos últimos casos de bosque mesófilo de montaña. En la literatura se menciona otro registro que amplía su distribución conocida a la India.

P. melastoma (Sow.: Fr.) Fuckel, fue citada por Pompa-González y Cifuentes (1991) de los estados de México y Guerrero, donde prospera en bosques de abetos, de pino-encino y de encino. Estas colectas constituyen una notable extensión al sur del área conocida de la especie, que se distribuye también en el oeste de Canadá y noroeste de Estados Unidos (Paden, 1983).

Sección Curvatisporae (esporas lisas y subalantoides)

P. campylospora (Berk.) Nannf. apud Korf, fue citada por Pompa-González y Cifuentes (1991) del estado de Guerrero, de bosque mesófilo de montaña. Este registro amplía su distribución para el neotrópico.

Sección Plicosporae (esporas elípticas con costillas transversales)

P. rhytidia fue citada por Paden (1983) para Colombia, Jamaica, Puerto Rico y México; en este último del estado de Veracruz, donde crece en bosque mesófilo de montaña. A pesar de que $P$. rhytidia f. rhytidia Donadini, ya se conocía del país, se creyó conveniente incluir su descripción, pues es poco común, además del apoyo que representan las fotografías al microscopio electrónico para separar esta forma de la platensis, citada como nuevo registro.

Es importante señalar que además de las seis especies que se discuten, se han excluido del presente trabajo a $P$. floccosa (Schw.) Seaver citada por Díaz-Barriga et al. (1988) de Michoacán y a $P$. occidentalis (Schw.) Sacc., registrada por Chacón y Bautista (1988) de Morelos, ya que actualmente tales especies se consideran como miembros de los géneros Microstoma y Sarcoscypha, respectivamente.

Plectania rhytidia f. platensis (Speg.) Donadini, Rivista Mycol. 28: 24. 1985. = Urnula platensis Speg., An. Mus. Nal. B. Aires II 3: 310. 1899. (Figs. 1, 4-9).

Apotecio de 0.7-18 mm de diámetro, en forma de copa, sésil, de color café obscuro, con la base rodeada de hifas gruesas, de color obscuro, que le sirven como anclaje. Superficie externa de color café-rojizo a casi negro, ligeramente más pálido en el himenio, marcado con prominentes costillas verticales, margen irregularmente crenulado.

Ascas de 171-188 x 11.2-13.6 $\mu \mathrm{m}$, cilíndricas, operculadas, inamiloides, de pared gruesa, octosporadas. Ascosporas de 20-24 x 9.6-12 $\mu \mathrm{m}$, elipsoides a subfusiformes, hialinas, con 9-11 estrías horizontales no anastomosadas, sólo presentes en la parte convexa de la espora. Paráfisis de $1.6 \mu \mathrm{m}$, filiformes, ramificadas hacia el ápice, inmersas en una matriz mucilaginosa. Pelos himeniales de 1.6-2.4 $\mu \mathrm{m}$ de diámetro, septados cerca de la base, de pared lisa. Excípulo medular de 300-550 $\mu \mathrm{m}$ de grosor, hifas intrincadas, 
Medel y Chacón: Contribución al Conocimiento del Género Plectania en México
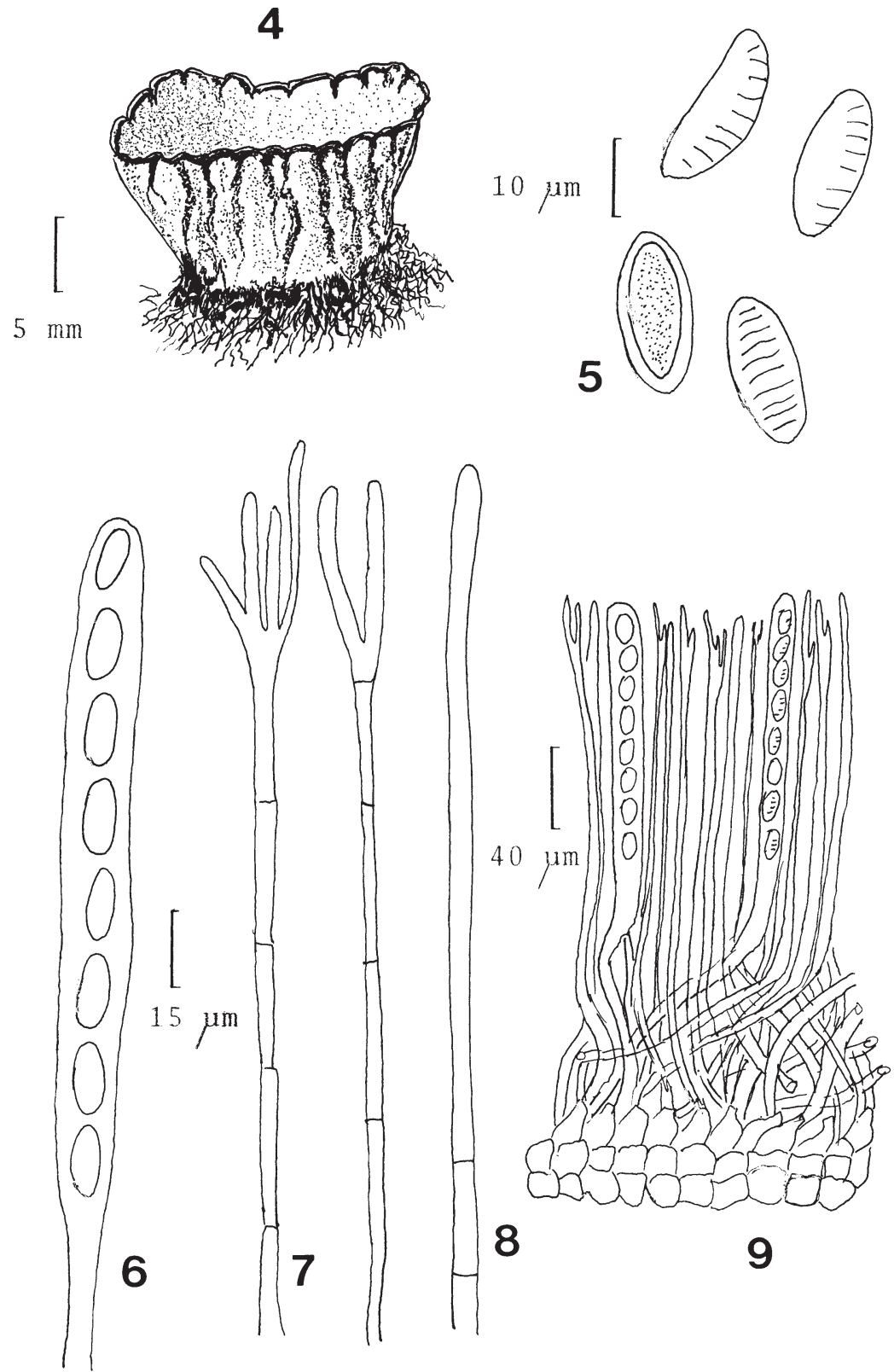

Figs. 4-9. Plectania rhytidia f. platensis. 4. apotecio; 5. ascosporas; 6. asca con ascosporas; 7. paráfisis; 8. pelos himeniales; 9. corte transversal del apotecio. 
de 1.6-2.4(4) $\mu \mathrm{m}$ de diámetro, septadas, hialinas a café obscuras. Excípulo ectal de 80$100 \mu \mathrm{m}$ de grosor, de textura angular a globosa, con células de 8-20(-30) x 8-11.2(-13) $\mu \mathrm{m}$, de color café obscuro a café pálido, las células más externas de color café obscuro.

Hábitat: Apotecios aislados a gregarios sobre corteza muerta de árboles en bosque mesófilo de montaña.

Material estudiado: VERACRUZ, Granja Santa Bárbara, km 10 NE de la carretera Xalapa-La Joya, municipio de Rafael Lucio, R. Medel 208, 246, 304; ibid., J. C. Anell 771; ibid., S. Chacón 3776; Jardín Botánico Francisco J. Clavijero, km 2.5 antigua carretera Xalapa-Coatepec, municipio de Xalapa, S. Chacón 3766, 4218 (XAL).

Discusión: Plectania rhytidia f. platensis se caracteriza por el color del apotecio y las esporas con estrías transversales no anastomosadas, según Rifai (1968), Otani (1973, 1980), Korf y Wen-Ying (1991), Paden (1983), Dennis et al. (1977) y Spegazzini (1899), quien describió este hongo como Urnula platensis. Es semejante a la forma rhytidia, de la cual se separa por el color café-rojizo del apotecio y por las esporas más grandes (18.4$24 \times$ 9.6-12 $\mu \mathrm{m}$ ) con estrías que parecen anastomosarse al microscopio de luz, sin embargo, las observaciones al microscopio electrónico mostraron menos de 11 estrías no anastomosadas. Otra diferencia consiste en el crecimiento solitario o gregario en la primera y definitivamente gregario en $P$. rhytidia f. rhytidia. Ambas formas se encontraron creciendo en bosque mesófilo de montaña. Se conoce de Argentina, Europa, África, Australia, así como de Japón y este es su primer registro para el país.

P. rhytidia (Berk.) Nannf. \& Korf f. rhytidia

= Plectania rhytidia (Berk.) Nannf. \& Korf, Mycologia 49: 110. 1957.

= Sarcosoma rhytidia (Berk.) Le Gal, Discom. Madag. 224. 1953. (Figs. 2, 3, 10-15).

Apotecio de 0.5-17 mm de diámetro, en forma de copa, sésil o subestipitado, de color café rojizo a obscuro, anclado al substrato por gruesos pelos negros. Superficie externa de color café obscuro, pared lisa a rugosa, con el margen ligeramente crenulado.

Ascas de 250-300 x 14.4-18 $\mu \mathrm{m}$, cilíndricas, operculadas, inamiloides, de pared gruesa, octosporadas. Ascosporas de 18-24 x 9.6-12 $\mu \mathrm{m}$, elipsoides a subfusiformes, hialinas, con 10-13 estrías horizontales aparentemente anastomosadas, las cuales cubren solamente la parte convexa de la ascospora. Paráfisis de $1.6 \mu \mathrm{m}$ de diámetro, cilíndricas, ramificadas basalmente, ápice simple o raramente ramificado, embebido en una matriz mucilaginosa. Pelos himeniales abundantes, de 1.6-2.4 $\mu \mathrm{m}$ de diámetro, ápice redondeado, septados hacia la base. Excípulo ectal de aproximadamente $85 \mu \mathrm{m}$ de diámetro, células angulares o isodiamétricas, de color café rojizo obscuro, de 9.6-12 $\mu \mathrm{m}$ diámetro, pared gruesa. Excípulo medular con hifas intrincadas de 2-3 $\mu \mathrm{m}$ de diámetro, de color café pálido a café obscuro hacia el excípulo ectal. Pelos superficiales de 4-8 $\mu \mathrm{m}$ de diámetro, negruzcos.

Hábitat: Apotecios gregarios, lignícolas sobre madera en descomposición en bosque mesófilo de montaña. 
Medel y Chacón: Contribución al Conocimiento del Género Plectania en México
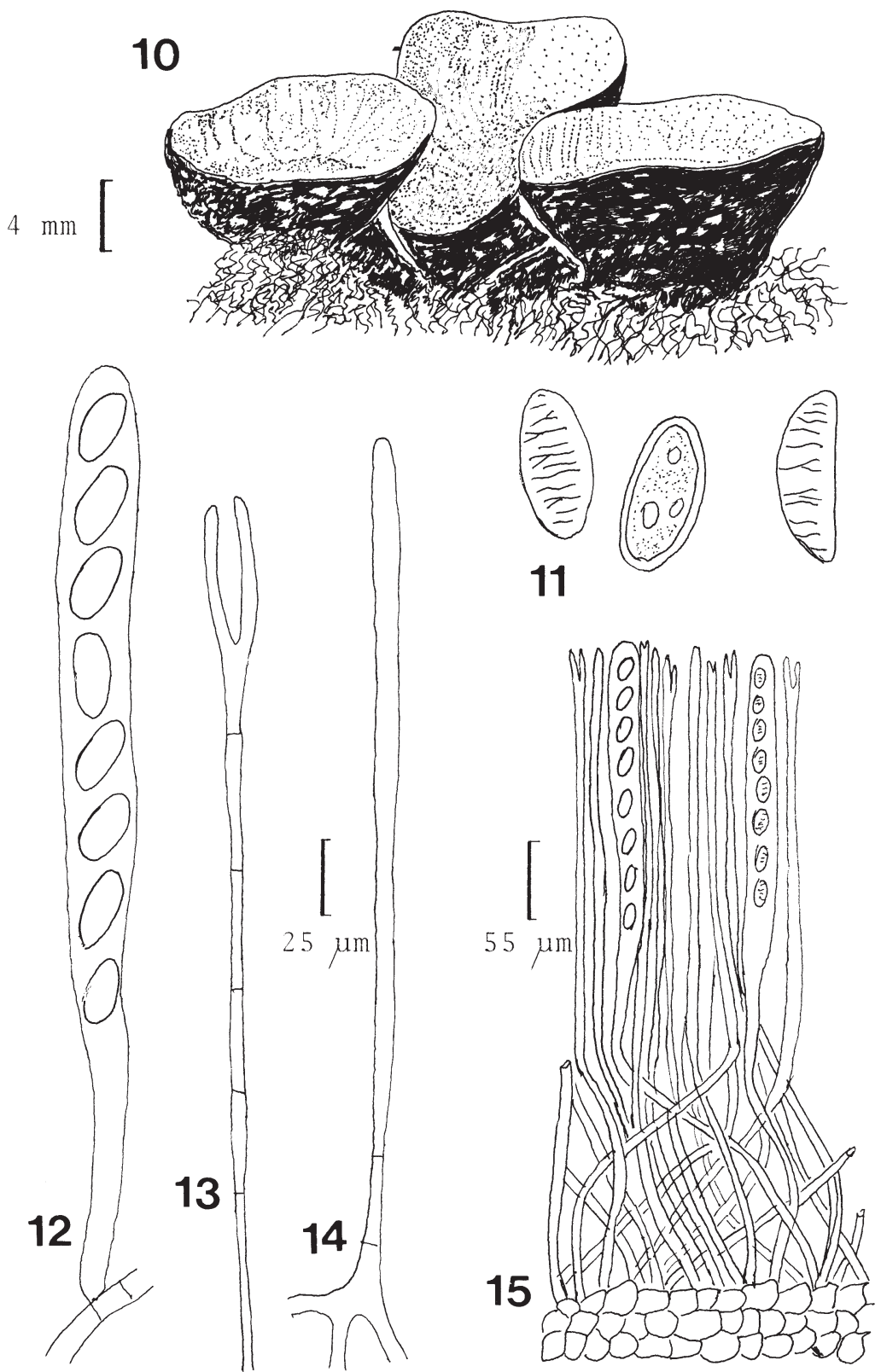

Figs. 10-15. Plectania rhytidia f. rhytidia. 10. apotecio; 11. ascosporas; 12. asca con ascosporas; 13. paráfisis; 14. pelos himeniales; 15. corte transversal del apotecio. 
Material estudiado: VERACRUZ, Granja Santa Bárbara km 10 NE de la carretera Xalapa-La Joya, municipio de Rafael Lucio, R. Medel 232 (XAL); ibid., J. García s. n., marzo 5, 1988 (XALUV). Jardín Botánico Francisco J. Clavijero, km 2.5 carretera antigua XalapaCoatepec, municipio de Xalapa, S. Chacón 1638 (XAL). Zamora, municipio de San Andrés Tlalnehuayocan, F. Ventura 13312 (XAL).

Discusión: $P$. rhytidia f. rhytidia se caracteriza por el color del apotecio y sus esporas con estrías que parecen anastomosarse al microscopio de luz. El material estudiado concuerda con las descripciones dadas por Rifai (1968), Korf y Wen-Ying (1991) y Le Gal (1953), quien se refirió a este hongo como Sarcosoma rhytidia (Berk.) Le Gal, aunque el material mexicano presenta ascas más cortas que las citadas en la literatura (de $550 \mathrm{x}$ 14.5-20 $\mu \mathrm{m}$, según Rifai (op. cit.) y de 425-570 x 16-22 $\mu \mathrm{m}$, según Le Gal (1953)). Al microscopio electrónico se observó que las esporas tienen estrías largas y cortas en número de 11 a 14, no anastomosadas. Esta forma es muy afín a la anteriormente descrita. Se conoce de Nueva Zelanda, Malasia y Madagascar.

\section{CLAVE PARA LA IDENTIFICACIÓN DE LOS REPRESENTANTES CONOCIDOS DE PLECTANIA EN MÉXICO}

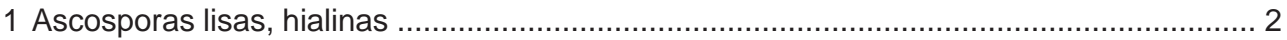

1 Ascosporas con estriaciones transversales, oliváceas ........................................... 5

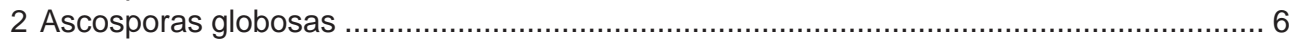

2 Ascosporas más largas que anchas .............................................................. 3

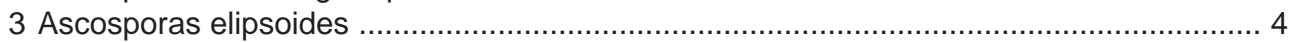

3 Ascosporas subalantoides .................................................................... P. campylospora

4 Apotecios pequeños, hasta de $5 \mathrm{~mm}$ de diámetro ..................................... P. melastoma

4 Apotecios grandes, hasta de $14 \mathrm{~mm}$ de diámetro ...................................... P. mexicana

5 Ascosporas con 9-11 estriaciones no anastomosadas ............... P. rhytidia f. platensis

5 Ascosporas con más de 11 estriaciones aparentemente anastomosadas

6 Apotecios sésiles.

P. rhytidia f. rhytidia

6 Apotecios estipitados

P. nigrella

P. melaena

\section{AGRADECIMIENTOS}

Los autores agradecen al Instituto de Ecología, A.C. y a la Facultad de Ciencias Forestales de Linares, N.L., por las facilidades para la realización de esta investigación. Al Dr. Gastón Guzmán se le reconocen los comentarios y sugerencias al trabajo. Uno de los autores (Medel) agradece al CONACyT la beca para la realización de sus estudios de maestría. Al Téc. Tiburcio Laez, del Instituto de Ecología, A.C. se le dan las gracias por la toma de las fotografías al microscopio electrónico. Los revisores anónimos contribuyeron a mejorar sustancialmente el artículo. 


\section{LITERATURA CITADA}

Chacón S. y N. Bautista. 1988. Ascomycetes poco conocidos de México, IV. Especies de Morelos, parte II. Biótica 13: 35-40.

Dennis, R. W. G., D. A. Reid y B. Spooner. 1977. The fungi of the Azores. Kew Bull. 32: 85-136.

Díaz-Barriga, H., F. Guevara y R. Valenzuela. 1988. Contribución al conocimiento de los macromicetos del estado de Michoacán. Acta Bot. Mex. 2: 21-44.

Frutis, I. y G. Guzmán. 1983. Contribución al conocimiento de los hongos del estado de Hidalgo. Bol. Soc. Mex. Mic. 18: 219-266.

Heredia, G. 1989. Estudio sobre los hongos de la reserva de la Biósfera El Cielo, Tamaulipas. Consideraciones sobre la distribución y ecología de algunas especies. Acta Bot. Mex. 7: 118.

Korf, R. P. 1957. Two bulgarioid genera: Galiella and Plectania. Mycologia 49: 107-111.

Korf, R. P. 1972. Synoptic key to the genera of the Pezizales. Mycologia 64: 937-994.

Korf, R. P. y Wen-Ying Zhuan. 1991. A preliminary discomycete flora of Macaronesia: part 11. Mycotaxon 40: $1-12$.

Le Gal, M. 1953. Les Discomycetes de Madagascar. Prodrome à une flore mycologique de Madagascar 4: $1-465$.

Otani, Y. 1973. On Pseudoplectania and Plectania collected in Japan. Rep. Tottori Mycol. Soc. Japan 21: $411-418$.

Otani, Y. 1980. Sarcoscyphineae of Japan. Trans. Mycol. Soc. Japan 21: 149-177.

Paden, J. W. 1983. Sarcosomataceae (Pezizales, Sarcoscyphineae). Flora Neotropica Monographs 37: $1-17$.

Paden, J. W. y E. Tilutky. 1969. Idaho Discomycetes. II. Mycologia 61: 683-693.

Pompa-González, A. y J. Cifuentes. 1991. Estudio taxonómico de los Pezizales de los estados de Guerrero, Hidalgo, Estado de México y Michoacán. Rev. Mex. Mic. 7: 87-112.

Rifai, M. A. 1968. The Australasian Pezizales in the Herbarium of the Royal Botanic Gardens Kew. Verh. Kon. Ned. Akad. Wetensch. Afd. Natuurk. 3: 1-295.

Spegazzini, A. 1899. Fungi Argentini novi. Anals. Mus. Nal. B. Aires. 6: 81-365.

Recibido en marzo de 1998. Aceptado en febrero de 2000. 